\title{
Hypothermia deaths in Jefferson County, Alabama
}

\author{
A J Taylor, G McGwin Jr, G G Davis, R M Brissie, T D Holley, L W Rue III
}

Center for Injury

Sciences, University of Alabama at

Birmingham

A J Taylor

G McGwin Jr

T D Holley

L W Rue

Section of Trauma, Burns, and Critical Care Medicine, Department of Surgery, School of Medicine, University of Alabama at Birmingham G McGwin Jr

L W Rue

Department of Epidemiology, School of Public Health, University of Alabama at Birmingham $\mathrm{G}$ McGwin Jr

Coroner/Medical Examiner Office, Jefferson County, Alabama and Department of Pathology, Forensic Division, University of Alabama at

Birmingham G G Davis

R M Brissie

Correspondence to: Gerald McGwin Jr, 120 Kracke Building, 1922 7th Avenue South, Birmingham, AL 35294-0016, USA mcgwin@eyes.uab.edu

Table 1 Hypothermia deaths/100 000 population in Fefferson County, Alabama, Fanuary 1983 to fuly 1999

\begin{tabular}{|c|c|c|c|c|c|c|c|}
\hline \multirow{2}{*}{$\begin{array}{l}\text { Age group } \\
\text { (years) }\end{array}$} & \multicolumn{3}{|l|}{ White } & \multicolumn{3}{|l|}{ Black } & \multirow[b]{2}{*}{ Total } \\
\hline & All & Male & Female & All & Male & Female & \\
\hline $0-9$ & 0.00 & 0.00 & 0.00 & 0.15 & 0.00 & 0.31 & 0.07 \\
\hline $10-19$ & 0.00 & 0.00 & 0.00 & 0.00 & 0.00 & 0.00 & 0.00 \\
\hline $20-29$ & 0.00 & 0.00 & 0.00 & 0.00 & 0.00 & 0.00 & 0.00 \\
\hline $30-39$ & 0.00 & 0.00 & 0.00 & 0.16 & 0.36 & 0.00 & 0.06 \\
\hline $40-49$ & 0.11 & 0.22 & 0.00 & 1.21 & 2.15 & 0.44 & 0.45 \\
\hline $50-59$ & 0.29 & 0.31 & 0.27 & 1.83 & 4.31 & 0.00 & 0.72 \\
\hline $60-69$ & 0.98 & 1.88 & 0.25 & 3.30 & 4.75 & 2.39 & 1.62 \\
\hline $70-79$ & 1.25 & 1.61 & 1.02 & 4.10 & 9.59 & 0.82 & 2.07 \\
\hline$>80$ & 1.24 & 1.53 & 1.13 & 13.35 & 16.93 & 10.08 & 4.61 \\
\hline Total & 0.28 & 0.37 & 0.19 & 1.12 & 1.65 & 0.68 & 0.66 \\
\hline
\end{tabular}

heat exposure. ${ }^{1}$ This finding may appear incongruous, given Alabama's southerly location and warm summer temperatures. Alabama's climate is characterized by long, hot summers and comparatively mild winters. The normal daily minimum temperatures for Birmingham in December, January, and February are $35.4,31.3$, and $34.5^{\circ} \mathrm{F}$, respectively. The record low, set in 1985, was -6 degrees. $^{2}$ Although exposure to hot environmental temperatures can result in serious illness, humans are better adapted to heat than they are to cold, ${ }^{3}$ and they readily acclimatise to hot weather over several weeks' time. ${ }^{3}$

A number of characteristics have been associated with an increased risk of hypothermia. The elderly are particularly susceptible to hypothermia, ${ }^{4-8}$ often suffering inadequate heat production because of reduced muscle mass or chronic disease. ${ }^{3}$ Alcohol consumption is also associated with hypothermia, as alcohol promotes heat loss through dilation of the superficial blood vessels and may impair judgment with respect to the need for adequate clothing and appropriate shelter. ${ }^{3}$ Other reported risk factors for hypothermia include male sex, ${ }^{4} 7910$ and black race. ${ }^{3}$ There is only limited information as to whether these factors are also associated with an increased risk of death from hypothermia.

In our previous study of hypothermia and hyperthermia deaths in Alabama we presented the demographic characteristics of hypothermia and hyperthermia deaths. ${ }^{1}$ Information on other factors that may have played a part in these deaths, such as the circumstances surrounding death and blood concentrations of alcohol and drugs, was not available from the dataset utilized, which was derived from death certificate data. In order to more thoroughly characterize hypothermia deaths in Alabama, we undertook the present study, which is based upon more detailed information abstracted from coroner's files. Of studies appearing in the published literature, only one other, which was conducted in Tokyo, ${ }^{11}$ has presented a comprehensive assessment of both demographic characteristics and circumstances surrounding hypothermia deaths. The purpose of this paper is to present the epidemiology of hypothermia deaths in Jefferson County, Alabama.

\section{Methods}

Coroner's files maintained by the Jefferson County, Alabama Coroner/Medical Examiner Office were reviewed for all cases with the primary or underlying cause of death recorded as "hypothermia" or "exposure to cold" occurring between January 1983 and July 1999. Information was abstracted from the files 


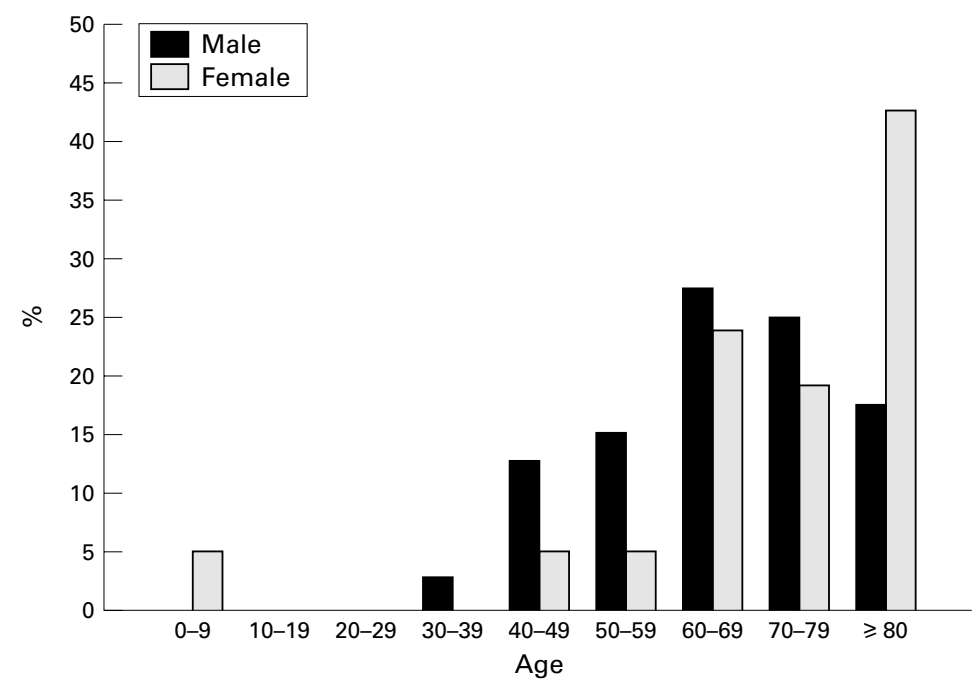

Figure 1 Age distribution of hypothermia deaths by sex population in Fefferson County, Alabama, fanuary 1983 to Fuly 1999.

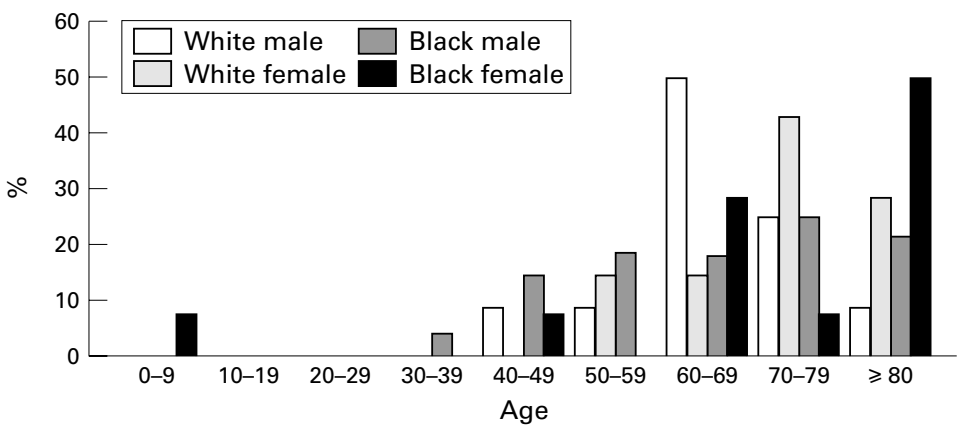

Figure 2 Age distribution of hypothermia deaths by race/sex group population in fefferson County, Alabama, Fanuary 1983 to fuly 1999.

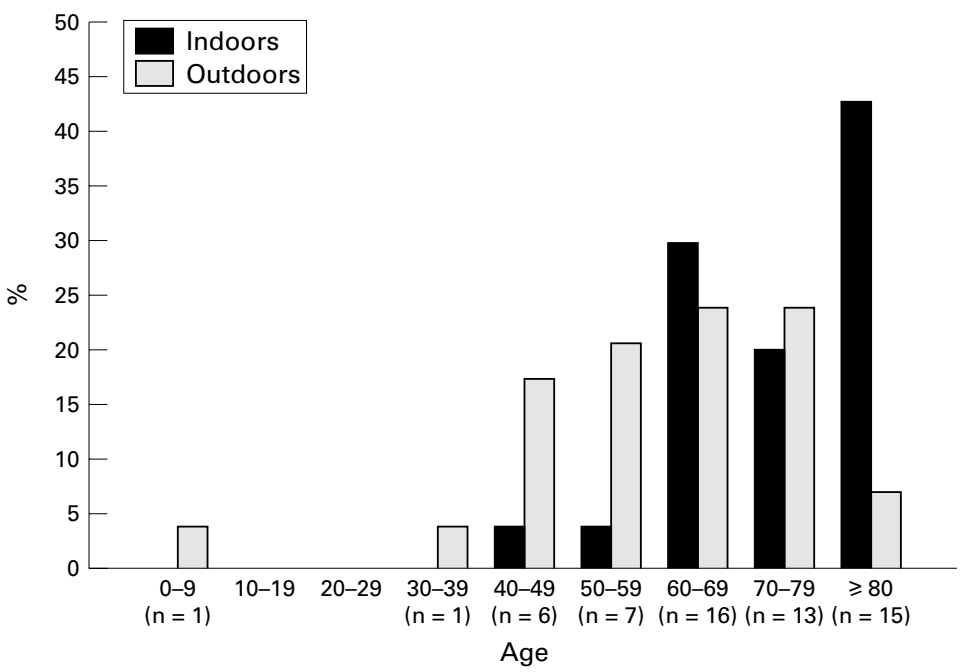

Figure 3 Age distribution by location of subject population in fefferson County, Alabama, fanuary 1983 to Fuly 1999.

including the extent of the postmortem examination (for example, full autopsy, external examination only, death certificate review only), age, race, sex, primary and underlying causes of death, blood alcohol concentration (BAC), other substances detected, location where the victim was found, circumstances of death, and underlying medical conditions. All variables were abstracted in cases of full autopsy or external examination. Information available in cases of death certificate review was less complete, with little or no information on circumstances of death, medical conditions, or toxicological findings.

\section{Results}

Sixty three hypothermia deaths occurred in Jefferson County, Alabama between January 1983 and July 1999, a rate 0.59/100 000 persons. The mean age of these deaths was 68 years (minimum: 5, maximum: 102). The highest rates of hypothermia death occurred among those aged 80 years or older (4.59/ 100 000), and, in general, rates increased with age (table 1). Overall, $63.9 \%$ of deaths were among males $(0.80 / 100000)$ and $70.0 \%$ were of black race $(0.73 / 100000)$. The age distributions of males and females were markedly different, female decedents tending to be older compared with their male counterparts (fig 1). Hypothermia deaths among individuals of black race exceeded those of whites for all age groups, markedly so among those aged 80 years and older (13.35/100 000 persons $v$ 1.24) (table 1). A similar pattern was observed when the decedents were stratified by race and sex (fig 2).

An approximately equal number of hypothermia deaths occurred indoors $(n=30)$ as outdoors $(n=29)$. However, deaths occurring indoors were more common among older persons while those occurring outdoors were more common among younger persons (fig 3). The majority of outdoor deaths occurred among blacks and males. Of those patients who died indoors, no evidence was recorded to suggest that their hypothermia had developed outdoors.

Ethanol was detected in the blood of $30 \%$ $(n=18$, excluding one death with a BAC of less than $0.01 \mathrm{~g} / \mathrm{dl}$ ) of hypothermia deaths. Nearly three quarters $(72.2 \%)$ of these deaths occurred outdoors. Detectable blood alcohol was reported among $50 \%$ of white males $(n=6), 0 \%$ of white females $(n=0), 35.7 \%$ of black males $(n=10)$, and $14.3 \%$ of black females $(n=2)$.

Nine of those individuals dying of hypothermia demonstrated detectable concentrations of drugs upon toxicological analysis. Two of the nine had detectable concentrations of phenobarbital, two of diazepam, one of nordiazepam, one of normeperidine, one of phenytoin, one of a tricyclic antidepressant, one of cocaine and cocaethylene, and one of pseudoephedrine. For the remainder of decedents, either no drugs were detected $(n=35)$ or toxicological analyses were not performed $(n=19)$.

The majority $(86.9 \%)$ of hypothermia decedents were identified as suffering from one or more chronic medical conditions (including alcoholism) at the time of death. Seventeen decedents $(27.0 \%)$ were reported to have histories of chronic alcoholism, four of whom were without detectable BACs upon analysis. If alcoholics (history of alcoholism or demonstrating both liver disease and blood alcohol) are excluded, the percentage of decedents with one or more chronic medical conditions is reduced to $52 \%$. Diabetes was reported as an 
underlying medical condition in three cases and a history of psychiatric illness in four cases.

\section{Discussion}

Hypothermia appears to be a greater problem in Alabama (and Jefferson County specifically) than in the United States in general. The rate of hypothermia death in Alabama is $0.43 / 100000$ population whereas the rate in the United States overall is $0.30 .^{1}$ The hypothermia death rate for Jefferson County is 0.59/100 000 .

Of hypothermia deaths in Jefferson County, Alabama between January of 1983 and July of 1999, the greatest percentage was among those of black race and the greatest percentage of those less than 80 years of age was among males. The highest rates of hypothermia death were among black males and among blacks 80 years of age or older. The majority of decedents suffered from one or more chronic medical conditions. Among decedents with detectable BACs $(30 \%)$, the majority occurred among black males and out of doors. Nine decedents had detectable blood concentrations of drugs or medication.

We observed that hypothermia deaths fell roughly into two categories: elderly persons found indoors and middle aged males found outdoors and having detectable BACs. Other studies have similarly noted these two groups among hypothermia cases presenting to a hospital emergency room ${ }^{12}{ }^{13}$ and among hypothermia deaths. ${ }^{11}$ Additional studies have reported findings of a greater number of hypothermia deaths among male subjects ${ }^{4910}$ and among subjects who had consumed alcohol. ${ }^{411}$ Older age has been associated with hypothermia in several studies. ${ }^{671014}$ Only the three studies cited above addressed whether decedents' bodies were found indoors or outdoors. ${ }^{1-13}$ Taylor et al divided decedents into "domestic" and "non-residential" deaths; however, both categories included indoor and outdoor deaths. ${ }^{14}$

We found the highest rate of hypothermia deaths among black males, followed by black females, white males, and white females. Findings were similar for other studies in which rates were reported. Black males consistently showed the highest rates of hypothermia death. Rango reported that in the United States nonwhite males had the highest rate of hypothermia death, followed by non-white females, white males, and white females. ${ }^{10}$ A study conducted in Washington, DC found the highest rate of hypothermia deaths among black males, followed by white males, black females, and white females. ${ }^{4}$ A study of the United States overall reporting hypothermia death rates according to sex only found males to have a rate 4.2 times that of females. ${ }^{6}$ In our study the death rate for males was 2.2 times that for females.

Although numbers of hypothermia deaths are relatively small, hypothermia is a preventable condition with a high case fatality ratio, particularly among the elderly. ${ }^{5}{ }^{14}$ Mortality reported in the literature for hypothermia ranges anywhere from $0 \%$ to greater than $50 \% .^{812}$ Several factors may be responsible for the elevated proportion of deaths among the elderly. ${ }^{16}$ The elderly are at particular risk both for developing hypothermia due to impaired thermoregulatory ability ${ }^{8171819}$ and temperature perception. ${ }^{9}$

Among decedents with detectable concentrations of alcohol present, 12 had BACs of 0.15 to $0.40 \mathrm{~g} / \mathrm{dl}$. (A BAC of $0.15 \mathrm{~g} / \mathrm{dl}$ or greater is thought to impair thermoregulatory ability. ${ }^{517}$ ) An additional six decedents had BACs of 0.08 to 0.13 , at or above the level considered in most states to be legally impaired for the purposes of driving a motor vehicle. Among the signs and symptoms manifested at a BAC of 0.07 or greater is impaired judgment. ${ }^{20}$ It may therefore be argued that although the individuals' thermoregulatory ability may not have been directly compromised by alcohol consumption, judgment may have been sufficiently impaired to prevent the individual from taking measures necessary to prevent hypothermia. Autopsy reports for these decedents frequently indicated a failure to seek shelter.

Some medications are reported to impair thermoregulatory ability. ${ }^{20}{ }^{21}$ Among the medications often implicated in hypothermia deaths are benzodiazepines. ${ }^{17}$ In our study, toxicological analysis revealed the presence of diazepam in two decedents and nordiazepam in one case. As detectable concentrations of drugs or medications were found among only nine decedents, drug use does not appear to have been a major contributor to overall mortality from this cause in Jefferson County.

The majority of hypothermia deaths occurred in individuals with one or more underlying chronic medical conditions. Chronic conditions, which may impair thermoregulatory function, ${ }^{22}$ have been reported as a risk factor for hypothermia. ${ }^{73}$ Kurtz reported that $10 \%$ of accidental hypothermia cases result in death, ${ }^{15}$ and when hypothermia is associated with an underlying medical condition, mortality increases to $75 \%-90 \% .{ }^{16}$ White found an overall mortality rate of $13.7 \%$ and a rate among patients with severe underlying illness of $42 \%$, and a particularly high rate among those with diabetes. ${ }^{13}$ Mental illness has been reported as a risk factor for development of hypothermia. ${ }^{1722}$

\section{LIMITATIONS}

A limitation of any study of hypothermia death is the presumptive nature of the assignment of this cause of death. A clinical diagnosis of hypothermia is based on the core body temperature. ${ }^{24}$ After death a diagnosis must be based upon circumstances surrounding the death, ${ }^{3}$ as the body temperature may continue to decrease between the time of death and the body's discovery and there is no anatomic finding that proves hypothermia. This adds a subjective component to assignment of the cause of death, which may result in either under or over-reporting of hypothermia deaths.

A limitation of autopsy data is the paucity of information on medical and social history. Homelessness, mental illness, poverty, alcoholism, starvation, and dehydration have been 
found to be risk factors for hypothermia. ${ }^{7}$ Markers of these conditions (for example, emaciation, liver disease, blood concentrations of prescription medications) may be evident upon autopsy or based upon the circumstances of death; however, it is not possible to develop an all inclusive personal history. Our ability to determine the existence of chronic medical conditions was limited to those described upon postmortem examination. The extent to which this resulted in underestimation of the prevalence is unknown. We were unable to evaluate the extent to which social factors, such as socioeconomic status or homelessness, contributed to hypothermia death. Hypothermia deaths may not represent a cross section of hypothermia cases, but rather a subset with a higher prevalence of risk factors associated with decreased survival.

\section{STRENGTHS}

A strength of this study is its reliance on coroner's data, which are more complete than data derived from death certificates. Coroner's files include a greater level of detail on circumstances of death, underlying medical conditions, and toxicological analyses than is generally available from death certificate data. The study by Tanaka et al, conducted in Tokyo, ${ }^{11}$ and a brief report on hypothermia deaths in Washington, DC were based on autopsy data. ${ }^{4}$ Other studies of hypothermia death have been based upon previously collected mortality data, largely death certificate data. Criteria for assigning hypothermia as a cause of death may not be uniform among jurisdictions, making comparisons difficult. Our data was obtained solely from the Jefferson County Medical Examiners/Coroner's Office, resulting in consistent assignment of hypothermia as a cause of death.

In this study, we found essentially two groups of individuals who died as a result hypothermia, elderly individuals found indoors and middle aged males found outdoors. This finding has been reported in studies conducted in other regions of the world. ${ }^{113}$ We had thought that climatic and cultural differences between those regions and Alabama might result in a different picture. We have suggested educational initiatives directed at both prevention of hypothermia and death from hypothermia. Further research is needed to evaluate whether hypothermia deaths and hypothermia patients are two distinct populations.

\section{Implications for prevention}

Hypothermia occurring inside a dwelling may be more of a problem in the south than in colder climates where longer cold spells necessitate a more constant winter heat source. Periods of extreme cold are typically of short duration in this region and residents are often inadequately prepared. The majority of indoor hypothermia deaths in our study occurred among the elderly. Educational interventions directed at the elderly should focus on prevention, recognition of symptoms, and the

\section{Key points}

- Jefferson County, Alabama has a higher rate of death from hypothermia than the United States overall.

- Hypothermia deaths occurred most frequently among elderly individuals found indoors and middle aged males with detectable alcohol concentrations found outdoors.

- Rates of death from hypothermia were greatest among black males and among individuals ages 80 years or older.

- Chronic medical conditions (including alcoholism) were present in nearly $90 \%$ of cases.

- Hypothermia is a preventable condition.

importance of seeking prompt medical treatment if hypothermia is suspected. Gautam et al reported that the elderly patients in their study had likely suffered prolonged hypothermia before being discovered by family members. ${ }^{25}$ Educational efforts should therefore be directed toward family members or other support persons in addition to the elderly themselves. These efforts might take the form of public service announcements televised during the winter months, similar in nature to the tornado safety messages aired in prone areas during storm season.

An intervention directed toward the middle aged male alcoholic group would be difficult to design and to implement. In urban locations, some educational messages might be disseminated via homeless shelters or soup kitchens, however, the prevalence of homelessness and poverty in this group is unknown, and shelters often bar individuals who appear to have consumed intoxicating beverages.

1 Taylor A, McGwin G. Temperature-related deaths in Taylor A, McGwin G. Temperature-
Alabama. South Med $\mathcal{f}$ 2000;93:787-92.

2 Birmingham Temperature and Rainfall Normals and Records. (Online) National Weather Service, Birmingham, Alabama <http://www.srh.noaa.gov/bmx/> (8 August 2000).

3 Lifschutz B, Donoghue E. Forensic pathology of heat- and cold-related injuries. Clin Lab Med 1998;18:77-90.

4 Centers for Disease Control and Prevention. Exposurerelated hypothermia deaths-District of Columbia, 19721982. MMWR Morb Mortal Wkly Rep 1982;31:669-71.

5 Centers for Disease Control and Prevention. Current trends hypothermia-United States. MMWR Morb Mortal Wkly Rep 1983;32(3):46-48.

6 Centers for Disease Control and Prevention. Perspectives in disease prevention and health promotion hypothermiaassociated deaths-United States, 1968-1980. MMWR Morb Mortal Wkly Rep 1985;34(50):753-4.

7 Centers for Disease Control and Prevention. Hypothermiarelated deaths-Vermont, October 1994-February 1996. related deaths-Vermont, October 1994-February 19
MMWR Morb Mortal Wkly Rep 1996;45(50):1093-5.

MMWR Morb Mortal Wkly Rep 1996;45(50):1093-5.
McAlpine C, Dell JLC. Outcome after episodes of McAlpine C, Dell JLC. Outcome after
hypothermia. Age Ageing 1987;16:115-18.

9 Macey S, Schneider D. Deaths from excessive heat and excessive cold among the elderly. Gerontologist 1993;33: 497-500.

10 Rango N. Exposure-related hypothermia mortality in the United States, 1970-79. Am F Public Health 1984;74:115960.

11 Tanaka M, Tokudome S. Accidental hypothermia and death from cold in urban areas. Int $\mathcal{F}$ Biometeorol 1991;32:242-6.

2 White J. Hypothermia: the Bellevue experience. Ann Emerg Med 1982;11:417-24.

13 Hislop L, Wyatt J, McNaughton G, et al. Urban hypothermia in the west of Scotland. BMF 1995;311:725-8.

14 Taylor N, Griffiths R, Cotter J. Epidemiology of Taylor N, Griffiths R, Cotter J. Epidemiology of
hypothermia: fatalities and hospitalizations in New Zeahypothermia: fatalities and hospitalization

15 Kurtz K. Hypothermia in the elderly: the cold facts. Geriatrics 1982;37:85-93. 
16 Danzl D, Pozos R, Auerbach P, et al. Multicenter hypothermia survey. Ann Emerg Med 1987;16:1042-55.

17 Kortelainen $M$. Drugs and alcohol in hypothermia and hyperthermia related deaths: a retrospective study. $\mathcal{F}$ Forensic Sci 1987:32:1704-12.

18 Collins K, Easton J, Exton-Smith A. Shivering thermogenesis and vasomotor responses with convective cooling in the elderly. F Physiol 1981;320:76P

19 Lloyd E. Hypothermia in the elderly. Med Sci Law 1988;28: 107-14.

20 Heat-related mortality in the United States, 1997. $7 A M A$ 1998;280:316-17.
21 Centers for Disease Control and Prevention. Heat-related illnesses and deaths-United States, 1994-1995. MMWR Tep 1995;44(25):465-8.

22 Ward M, Cowley R. Hypothermia: a natural cause of death. Am F Forensic Med Pathol 1999;20:383-6.

23 Thomas D. Accidental hypothermia in the sunbelt. 7 Gen Intern Med 1988;3:552-4.

24 Miller J, Danzl D, Thomas D. Urban accidental hypothermia: 135 cases. Ann Emerg Med 1980;9:456-61.

25 Gautam P, Ghosh S, Madal A, et al. Hypothermia in the elderly: sociomedical characteristics and outcome of 86 patients. Public Health 1989;103:15-22.

\section{Editorial board member: brief biography}

JOHN LANGLEY

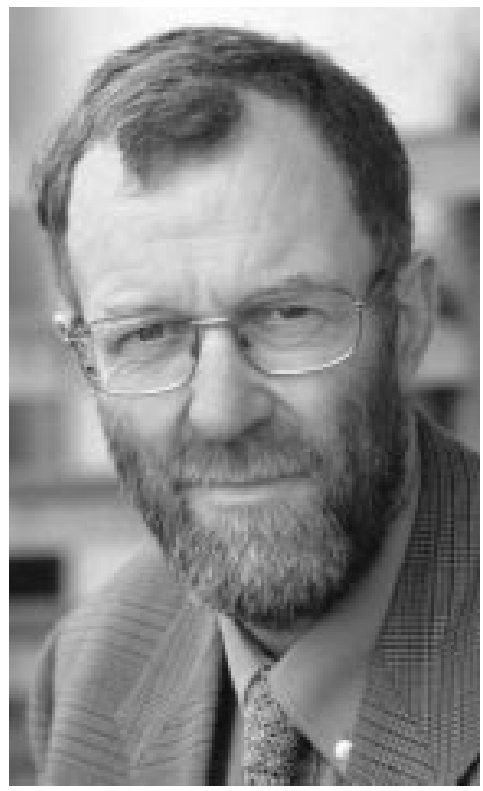

John has been involved in injury prevention research since 1978. His initial research focused on the Dunedin Multidisciplinary Health and Development Study, a longitudinal study of the health and development of a thousand children. Research based on that study formed the basis for his $\mathrm{PhD}$ from the University of Otago, conferred in 1985. In 1987 Dr Elizabeth McLouglin and he were commissioned by the Health Research Council of New Zealand (HRC) and Accident Compensation Corporation (ACC) to undertake a major review of injury prevention research in New Zealand. The recommendations in that review have played a key part in the development of injury prevention research in New Zealand.

With Associate Professor David Chalmers, he was successful in tendering to HRC and ACC for the establishment of the Injury Prevention Research Unit (IPRU). He has been the Director since its inception in 1990. The IPRU has been a focal point for injury prevention research in New Zealand ever since.

John has published more than 150 refereed papers plus numerous other publications dealing with a wide range of prevention issues including: childhood injury, motor vehicle traffic crashes, suicide, injury to Maori, injury surveillance, firearm injury, fireworks, assault, burns, drowning, and work related injury. He has been a strong advocate for legislative, policy, and practice changes aimed at reducing injury in these and other areas. 